\title{
Racial and Ethnic Disparities in Breastfeeding
}

\author{
Katherine M. Jones, ${ }^{1,2}$ Michael L. Power, John T. Queenan, ${ }^{3}$ and Jay Schulkin ${ }^{1}$
}

\begin{abstract}
This article's aim is to review the literature on racial and ethnic disparities in breastfeeding rates and practices, address barriers to breastfeeding among minority women, conduct a systematic review of breastfeeding interventions, and provide obstetrician-gynecologists with recommendations on how they can help increase rates among minority women. In order to do so, the literature of racial and ethnic disparities in breastfeeding rates and barriers among minority women was reviewed, and a systematic review of breastfeeding interventions among minority women on PubMed and MEDLINE was conducted. Racial and ethnic minority women continue to have lower breastfeeding rates than white women and are not close to meeting the Healthy People 2020 goals. Minority women report many barriers to breastfeeding. Major efforts are still needed to improve breastfeeding initiation and duration rates among minority women in the United States. Obstetrician-gynecologists have a unique opportunity to promote and support breastfeeding through their clinical practices and public policy, and their efforts can have a meaningful impact on the future health of the mother and child.
\end{abstract}

\section{Introduction}

$\mathbf{I}$

T IS WELL ESTABLISHED THAT breastfeeding is beneficial for the mother, baby, and society; however, the proportion of mothers breastfeeding in the United States is disappointing. ${ }^{1}$ Recognizing the importance of breastfeeding, the U.S. Department of Health and Human Services sets forth national breastfeeding objectives for women every decade. The Healthy People 2010 initiative set the following goals for breastfeeding: $75 \%$ of all mothers to initiate breastfeeding, with $50 \%$ continuing for at least 6 months postpartum and $25 \%$ continuing to 1 year as well as $40 \%$ exclusively breastfeeding at 3 months and $17 \%$ exclusively at 6 months. The only national objective for Healthy People 2010 that was met was that $75 \%$ of new mothers initiated breastfeeding. ${ }^{2}$ However, national rates of continued breastfeeding at 6 and 12 months and rates of exclusive breastfeeding at 3 and 6 months fell well below public health recommendations (Table 1).

Based on the more ambitious Healthy People 2020 objectives, the gap has grown even wider between breastfeeding outcomes and Centers for Disease Control and Prevention goals (Table 1). Asian women are currently the only racial/ ethnic group meeting the Healthy People 2020 goal of breastfeeding initiation of $81.9 \%$, although Hispanic women are very close, with certain subgroups of Hispanic women meeting this objective. ${ }^{3}$ African American women have the lowest rates of breastfeeding initiation, as well as continuation at 6 months and 12 months, compared with all other racial/ethnic groups in the United States. No racial/ethnic group is currently meeting the Healthy People 2020 objectives for continued breastfeeding at 6 months (61\%) and 12 months (34\%) and exclusive breastfeeding at 3 months (46\%) and 6 months $(26 \%){ }^{3}$

Mothers with lower rates of breastfeeding tend to be young, low-income, African American, unmarried, less educated, participants in the Supplemental Nutrition Program for Women, Infants, and Children (WIC), overweight or obese before pregnancy, and more likely to report their pregnancy was unintended. ${ }^{4-7}$ These final two points are important to highlight because African American and Hispanic women have the highest rates of being overweight or obese and the largest number of unintended pregnancies. ${ }^{8}$

\section{The Importance of Breastfeeding for Minority Women}

Breastfeeding is beneficial to almost all mothers and infants, but the benefits may be significantly greater for minority women. ${ }^{9}$ Minority women are disproportionately affected by adverse health outcomes, which may improve with breastfeeding. ${ }^{10}$ Relative to white women in the United States, African American and Hispanic women have increased rates of obesity, diabetes, and cardiovascular disease. ${ }^{11,12}$ Women who breastfeed exclusively are more likely to lose weight

\footnotetext{
${ }^{1}$ Department of Research, American College of Obstetricians and Gynecologists, Washington, D.C.

${ }^{2}$ Department of Psychology, American University, Washington, D.C.

${ }^{3}$ Department of Obstetrics and Gynecology, Georgetown University School of Medicine, Washington, D.C.
} 
Table 1. Healthy People 2010 and 2020 Goals and Centers for Disease Control and Prevention DATA From 2007 on Racial and Ethnic BREASTFEeding INITIATION AND Continuation ${ }^{2,3}$

\begin{tabular}{lrccc}
\hline & & \multicolumn{3}{c}{ Breastfeeding } \\
\cline { 4 - 5 } Racelethnicity & $\mathrm{n}$ & Ever & At 6 months & At 12 months \\
\hline Healthy People 2010 Goals & & $75 \%$ & $50 \%$ & $25 \%$ \\
American Indian or Alaska Native & 552 & $73.8 \pm 6.9$ & $42.4 \pm 8.8$ & $20.7 \pm 7.0$ \\
Asian or Pacific Islander & 1,077 & $83.0 \pm 5.2$ & $56.4 \pm 6.3$ & $32.8 \pm 6.5$ \\
$\quad$ Asian & 886 & $86.4 \pm 5.7$ & $58.6 \pm 7.1$ & $34.8 \pm 7.5$ \\
$\quad$ Native Hawaiian and other & 239 & $72.4 \pm 11.1$ & $45.3 \pm 12.1$ & $23.9 \pm 10.8$ \\
African American & 2,606 & $59.7 \pm 2.9$ & $27.9 \pm 2.5$ & $12.9 \pm 1.9$ \\
White & 13,425 & $77.7 \pm 1.2$ & $45.1 \pm 1.5$ & $23.6 \pm 1.3$ \\
Hispanic & 2,895 & $80.6 \pm 2.4$ & $46.0 \pm 3.1$ & $24.7 \pm 2.8$ \\
Healthy People 2020 goals & & $81.9 \%$ & $61 \%$ & $34 \%$ \\
\hline
\end{tabular}

postpartum than women who breastfeed nonexclusively and formula-feed. ${ }^{13,14}$ Some studies also suggest that breastfeeding may decrease the risk for obesity in the infant during childhood. ${ }^{15}$ Longer breastfeeding duration has also been associated with reduced incidence of type 2 diabetes in the mother. ${ }^{16}$ Research suggests that breastfeeding may reduce a mother's risk of hypertension, hyperlipidemia, and cardiovascular disease. ${ }^{17,18}$ Lastly, minority women have greater rates of unintended pregnancy. ${ }^{19}$ Exclusive breastfeeding on a strict, routine schedule can reduce fertility by inducing amenorrhea, thus delaying ovulation and increasing the interval between offspring. ${ }^{20}$

\section{Racial and Ethnic Disparities in Breastfeeding}

\section{African American women}

African Americans continue to have the lowest rates of breastfeeding initiation $(60 \%)$ and continuation at 6 months (28\%) and 12 months (13\%) compared with all other racial/ ethnic groups in the United States. ${ }^{3}$ Although improvements in breastfeeding rates for African American women are evident from the 2000-2007 National Immunization Survey (Figs. 1 and 2), African American mothers are still 2.5 times less likely to breastfeed than white women. ${ }^{21}$ A 16 percentage-point gap in the prevalence of continued breastfeeding
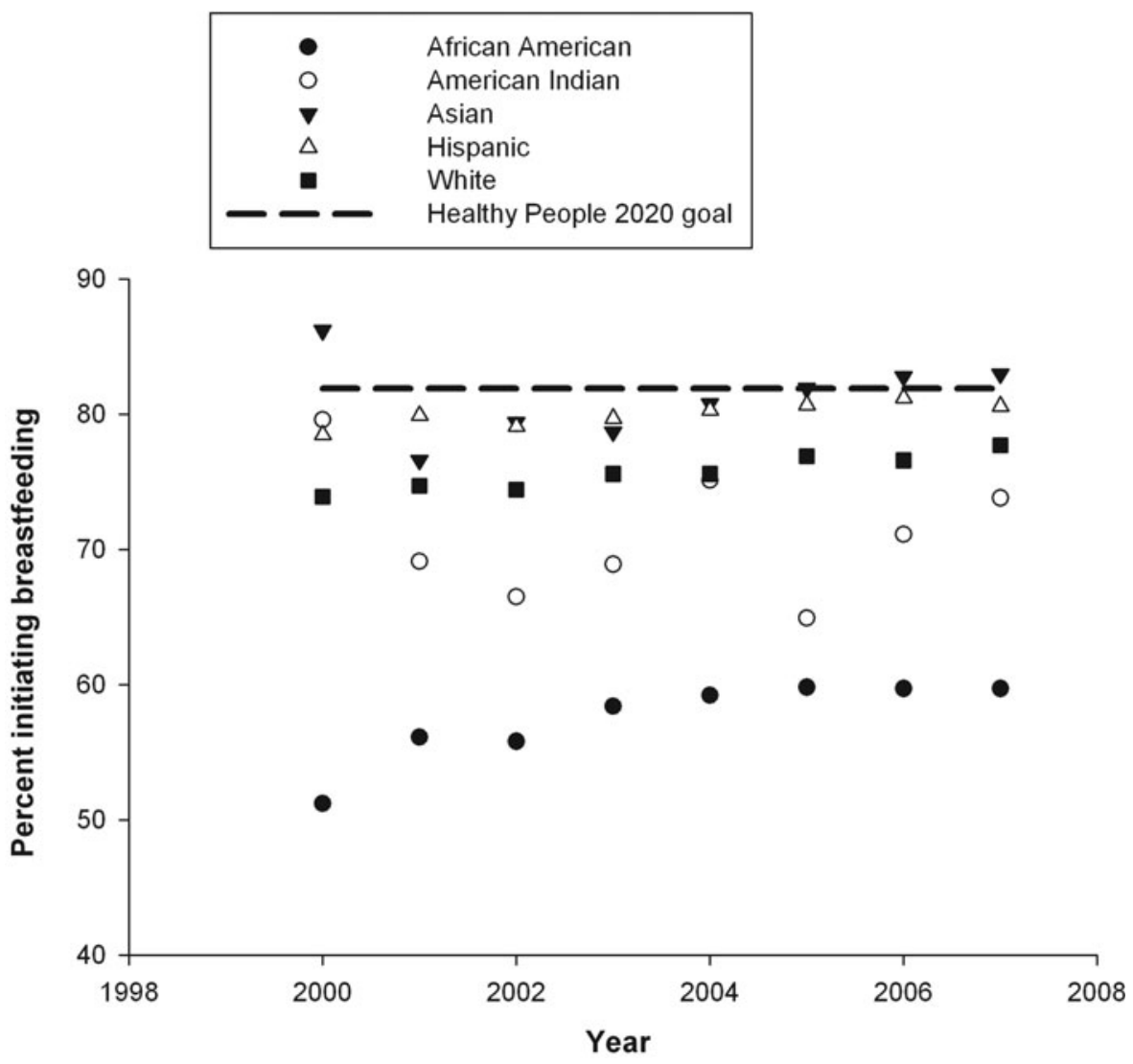

FIG. 1. Rates of initiation of breastfeeding by race/ethnicity from 2000 to 2007. 


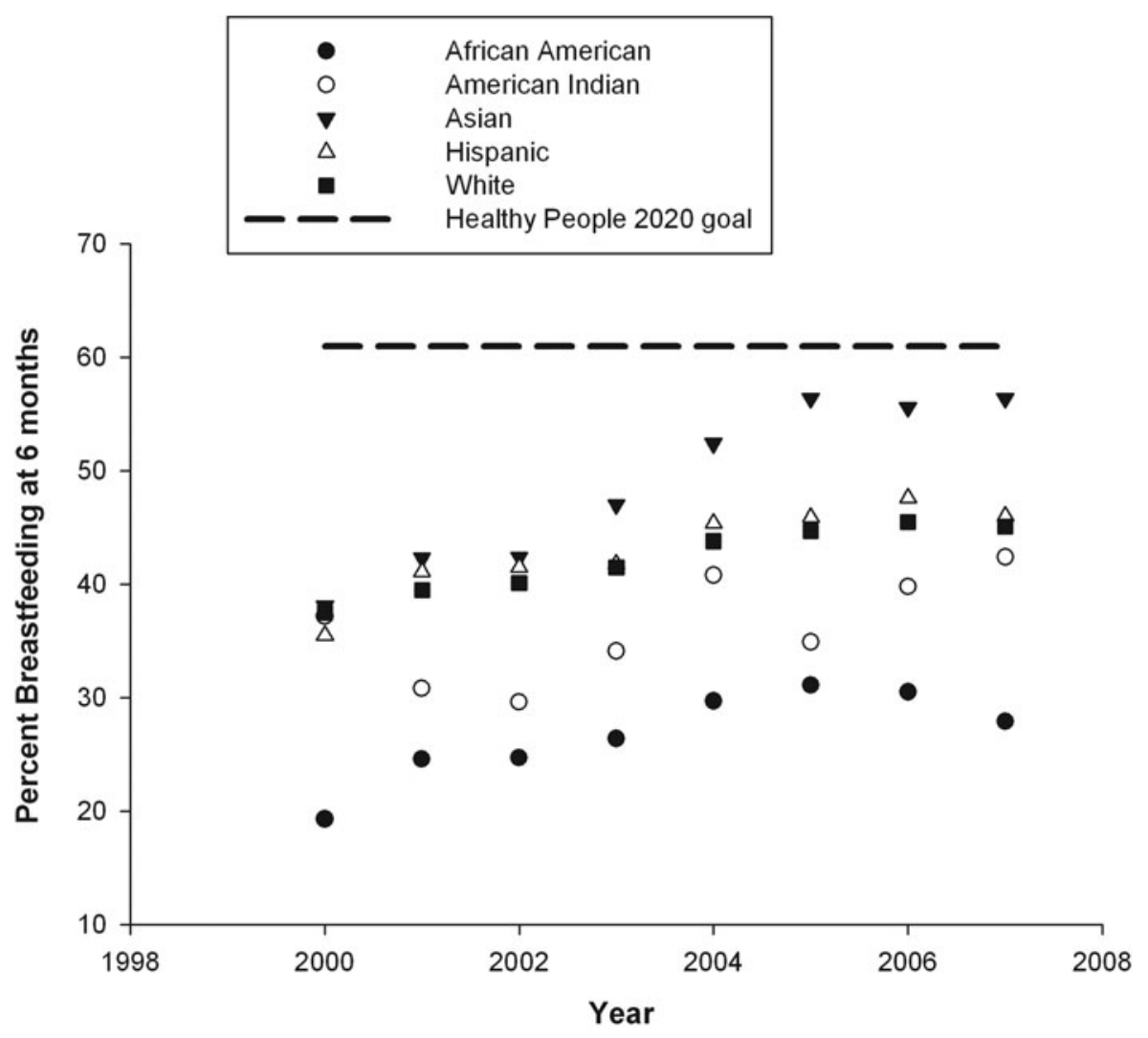

FIG. 2. Rates of breastfeeding at 6 months by race/ethnicity from 2000 to 2007.

for 6 months has been consistent since 1990 between African American and white women. ${ }^{2}$ African American women $(32 \%)$ are also more likely than most minority groups to provide formula supplementation by 2 days of life. ${ }^{22}$ Currently, African American women are not meeting any of the Healthy People 2020 objectives for breastfeeding (Table 1).

\section{Hispanic women}

Although Hispanic women have the highest rates of breastfeeding initiation and continuation among all women in the United States, there remain some ethnic health disparities among Latina mothers and children that are worth noting. ${ }^{3}$ Hispanic mothers are not presently meeting Healthy People 2020 objectives for postpartum breastfeeding continuation and exclusive breastfeeding. ${ }^{22}$ Hispanic mothers (33\%) are slightly more likely than other racial/ethnic groups to provide formula supplementation as early as 2 days of life. ${ }^{22}$ Compared with white women, Hispanic and African American mothers are more likely to introduce solid foods before 4 months of age, have higher rates of maternal restrictive feeding practices, and lower rates of exclusive breastfeeding. ${ }^{23}$

There is evidence for significant variation in breastfeeding practices among ethnic subgroups of Hispanic women. However, U.S. national data do not typically report breastfeeding rates for individual subgroups, which can mask large between-group variation. ${ }^{24}$ For example, breastfeeding rates are significantly higher for Mexican Americans than for Puerto Ricans. ${ }^{25}$ Chapman et al. ${ }^{26}$ found significantly lower rates of breastfeeding continutation among Puerto Ricans (median breastfeeding duration, <0.5 months) compared with Hispanic women from other Spanish-speaking countries (median breastfeeding duration, > 6.0 months). Moreover, differences in breastfeeding patterns have been noted among Hispanic women living in western versus eastern states. Compared with white women, Hispanic women have lower rates of breastfeeding when living in western states but have higher rates when living in eastern states. ${ }^{2}$

Another major factor impacting Hispanics' decision to breastfeed is their level of acculturation. There has been some disagreement within the literature regarding the association between acculturation and breastfeeding practices among Hispanic women. Beck $^{27}$ reviewed the perinatal literature from 1990 to 2005 on the association between acculturation and breastfeeding among Hispanic women. Several studies examining this association found a negative relationship between acculturation and likelihood of breastfeeding. ${ }^{28-31}$ Rassin et al. ${ }^{28}$ found that least acculturated Mexican Americans had the highest rates of breastfeeding initiation $(52.9 \%)$, whereas those who were most acculturated exhibited the lowest rates of breastfeeding initiation $(36.1 \%)$. Several other studies have also found evidence that Hispanic breastfeeding rates decrease with greater acculturation. ${ }^{29-31}$ Anderson et al. ${ }^{32}$ did not find any association between level of acculturation and breastfeeding rates among Puerto Rican women relative to Mexican American women living in the United States. The authors explained that their results could be due to differences in breastfeeding background and culture, in addition to varying breastfeeding support systems in the country of origin.

These results provide further evidence that Hispanic subpopulations are notably heterogeneous. Rates for exclusive 
breastfeeding at 6 months are $97 \%$ in Chile, $96 \%$ in Ecuador, 95\% in Colombia, 59\% in Bolivia, 38\% in Mexico, and $10 \%$ in the Dominican Republic. ${ }^{33}$ Breastfeeding attitudes are often carried over to the United States when Latinas immigrate, and rates among these women living in the United States may be strongly influenced by the background rate in their country of origin.

\section{American Indian/Alaska Native women}

The current literature on breastfeeding patterns among American Indian/Alaska Native (AI/AN) mothers is scarce, thus warranting further research. Based on the limited data, AI/AN mothers have lower rates of breastfeeding initiation, duration, and exclusivity relative to other racial/ ethnic groups except for African Americans. ${ }^{3}$ Apart from African American women, breastfeeding duration and exclusivity rates decline faster among AI/AN women than among other racial/ethnic groups. ${ }^{3}$ AI/ANs have the second lowest rates of breastfeeding initiation (73.8\%) among all races/ethnicities following African American women (59.7\%), as well as the second lowest rates of breastfeeding duration relative to African American women at 6 months (42.4\% and $27.9 \%$, respectively) and 12 months (20.7\% and $12.9 \%$, respectively). ${ }^{3}$ Rates for breastfeeding initiation and duration among $\mathrm{AI} / \mathrm{AN}$ women still require much improvement in order to meet the Healthy People 2020 goals.

Lindberg et al. ${ }^{34}$ found that among $\mathrm{AI} / \mathrm{AN}$ mothers, rates of breastfeeding initiation were low (59\%), and those of formula supplementation were high (97\%). Results also indicated that $76 \%$ of AI/AN mothers terminated breastfeeding within 4 months of the child's birth. These rates are significantly higher than typically observed in U.S. samples. ${ }^{35}$ Furthermore, their results indicated that among AI/AN mothers who initiated breastfeeding, the median duration was only 13 weeks, and the median time for introducing solid foods was 22 weeks. On a positive note, AI/AN mothers who were still breastfeeing at 6 months were more likely to still be breastfeeding at 12 months. ${ }^{36}$ Although fewer than half of all AI/AN mothers who initiated breastfeeding were still breastfeeding at 4 months, they were among several other racial/ethnic groups with the highest rate of breastfeeding at 12 months.

\section{Asian women}

Overall, Asian mothers have high breastfeeding initiation rates and are currently meeting the Healthy People 2020 goals. ${ }^{3}$ At 6 and 12 months, Asian mothers have the highest breastfeeding rates relative to all other racial/ethnic groups; however, there are some disparities among Native Hawaiian and Filipino women. ${ }^{37}$ Although the breastfeeding initiation rate in Hawaii for the general population $(89.6 \%)$ is higher than the national average, Native Hawaiian women (64\%) have much lower breastfeeding initiation rates. ${ }^{37}$ The percentage of mothers who continue breastfeeding for at least 6 months in Hawaii is above the national average; however, only $25 \%$ of Native Hawaiian mothers who initiated breastfeeding are still breastfeeding exclusively at 20 weeks. ${ }^{37}$ Furthermore, Native Hawaiian mothers introduce formula at a very early age, with $46 \%$ introducing formula within the first week after delivery. ${ }^{37}$

\section{Barriers to Breastfeeding Among Minority Women}

There are numerous barriers to breastfeeding that affect all women such as reported pain/discomfort, embarrassment, employment, and inconvenience. However, there are also barriers that are unique and more frequent among racial/ ethnic minority women. Major barriers to breastfeeding reported by low-income minority women include lack of social, work, and cultural acceptance/support, language and literacy barriers, lack of maternal access to information that promotes and supports breastfeeding, acculturation, and lifestyle choices, including tobacco and alcohol use (Table 2 gives a comprehensive list). ${ }^{5,21,38-40}$ One of the largest studies that has examined the impact of nativity/immigrant status, race/ ethnicity, and socioeconomic factors on breastfeeding in the United States found that immigrant women in each racial/ ethnic group had significantly higher rates of breastfeeding initiation and duration than native women. ${ }^{31}$ Immigrant children with foreign-born parents had the highest likelihood of being breastfed at 6 and 12 months, whereas the chances of not being breastfed at 6 and 12 months were twice as high among native children with native parents.

Barriers that affect African American and Hispanic women include historical, cultural, social, economic, political, and psychosocial factors. It is important to recognize the historical challenges that African American women have faced, which may have shaped perceptions and attitudes toward breastfeeding in the African American community and subsequently contributed to lower breastfeeding rates among these women.

Several studies have found that WIC participation is strongly associated with low rates of breastfeeding initiation and early breastfeeding discontinuation, particularly among African American and Hispanic women. ${ }^{36,41}$ The Centers for Disease Control and Prevention reports that the breastfeeding rate among low-income women participating in WIC is $67.5 \%$, whereas the rate for higher income WIC-eligible participants is $84.6 \% .^{2} \mathrm{Beal}$

Table 2. Common Barriers to Breastfeeding for Mothers, Which May Be Particularly Relevant FOR RACIAL AND ETHNIC MinORITY WOMEN

- Preference to bottle feed

- Pain

- Discomfort

- Lack of social and cultural acceptance

- Employment

- Inconvenience

- Lack of time

- Lack of parental knowledge regarding breastfeeding practices

- Lack of maternal access to information that promotes and supports breastfeeding

- Sexual perceptions

- Language and literacy barriers

- Acculturation

- Lifestyle choices, including smoking, alcohol use, and the desire of younger women who want to be independent and free to leave their infant in the care of others

- Lack of support from family, peers, work, and the healthcare community

- Difficulty with the baby latching onto the breast

- Inadequate milk production 
et al. ${ }^{42}$ found that African American women were less likely than white women to report having received breastfeeding advice from WIC counselors and, instead, were more likely to report having received formula-feeding advice. WIC counselors may have a significant impact on African Americans' and Hispanics' decisions to breastfeed given that African American women (19.2\%) and Hispanic women (39.2\%) make up the largest minority proportion of the total population of WIC women. ${ }^{43}$ The U.S. Department of Agriculture reports that although the racial/ethnic makeup for WIC's certification categories mirrors the overall racial/ethnic distribution of WIC clients, there is one noteworthy exception. Breastfeeding women in WIC are disproportionately Hispanic relative to all WIC women: $49.4 \%$ of breastfeeding women are Hispanic, whereas $39.2 \%$ of all WIC women are Hispanic. African American women (15.2\%) represent a smaller proportion of all breastfeeding women in WIC. $^{43}$

In addition to the possible disincentive to breastfeed due to access to free infant formula, low breastfeeding rates specifically among African American mothers may also reflect a negative perception of breastfeeding in the African American community. ${ }^{44}$ Another frequently reported barrier by African American women is lack of access to information that promotes and supports breastfeeding. ${ }^{39}$ In a study by Kulka et al., ${ }^{39}$ African American mothers reported that they need more specific information about what to expect and how to address possible complications during breastfeeding. Supporting previous findings, these mothers voiced concerns about differential treatment from healthcare providers with regard to breastfeeding encouragement and information. These results are particularly troubling because it is well documented that women who are encouraged by healthcare professionals are more likely to initiate breastfeeding. ${ }^{45}$

Another frequently cited barrier to breastfeeding initiation and continuation by low-income minority women is the necessity to return to work. ${ }^{4}$ Working breastfeeding women face conflict in balancing the ability to breastfeed with the demands of work (e.g., inflexible hours, decreased income, and limited maternity leave). ${ }^{46}$ Many low-income jobs are not covered under the Family and Medical Leave Act, forcing low-income women to return to work sooner than other women and possibly before breastfeeding is well established. ${ }^{47}$ The majority of women in the United States return to work between 3 and 6 months after birth; however, African American women typically return to work 2 weeks earlier and are more likely to have jobs that are not welcoming to breastfeeding. ${ }^{48}$ It is important to be sensitive to the challenges working women may face, while providing them with information about the benefits of breastfeeding as well as possible strategies and support systems to encourage continuing to feed their babies on breast milk.

\section{Breastfeeding Interventions: A Systematic Review}

Several studies have identified successful interventions to increase breastfeeding rates among racial/ethnic minority women. Chapman and Pérez-Escamilla ${ }^{22}$ conducted a systematic review of PubMed searches to evaluate the current status of knowledge regarding the effectiveness of breastfeeding interventions targeting minority women. They selected 22 articles that evaluated 18 interventions: peer counseling (PC) $(n=4)$, professional support $(n=4)$, a breastfeeding team (peer + professional support; $n=3$ ), breastfeeding-specific clinic appointments $(n=2)$, group prenatal education $(n=3)$, and enhanced breastfeeding programs $(n=2)$. Results indicated that PC interventions (alone or in combination with a health professional), breastfeeding-specific clinic appointments, group prenatal education, and hospital/WIC policy changes significantly improved breastfeeding initiation, duration, or exclusivity. Postpartum breastfeeding support offered by nurses was found to be the least effective intervention.

Because Chapman and Pérez-Escamilla ${ }^{22}$ recently conducted a systematic review of breastfeeding interventions targeting racial/ethnic minority women, we used their review as a foundation for ours. We conducted a systematic review of PubMed and MEDLINE searches in December 2013 to determine whether there were other relevant studies that had not been included in their review. Like Chapman and PérezEscamilla, ${ }^{22}$ we used "breastfeeding" and "randomized trial" along with each of the following descriptors "Latina," "Latino," "Hispanic," "black," "African American," “Asian," "Native American," "First Nation," "Indian," "minority," and "low-income." We also included the descriptor "American Indian," which was not included in the previous review. Furthermore, we conducted another search using "breastfeeding," "randomized trial," and "intervention" along with each of the descriptors listed above. Lastly, we conducted a search using "breastfeeding interventions among minority women" and "breastfeeding interventions among low-income women." We used the same inclusion criteria as Chapman and Pérez-Escamilla ${ }^{22}$ : studies had to be U.S.-based randomized controlled trials evaluating a breastfeeding promotion intervention, enrolling primarily racial/ethnic minority women, reporting breastfeeding outcome data, and conducting analyses with intention to treat. We only included studies that were published after 1999. After relevant abstracts were identified, the full text article was reviewed to identify those meeting inclusion criteria.

Based on the main focus of the intervention and the ways in which Chapman and Pérez-Escamilla ${ }^{22}$ categorized their results, the studies included in our review were grouped into one of the following three categories: PC, professional support, and enhanced breastfeeding programs (program addons). In regard to the studies included in our review, breastfeeding initiation indicates that the infant was ever breastfed or received breast milk. Exclusive breastfeeding reflects that the infant received only breast milk, allowing for vitamin/ mineral drops and medications, in accordance with the World Health Organization definition of exclusive breastfeeding. ${ }^{49}$

Our search yielded seven studies that were not included in the systematic review by Chapman and Pérez-Escamilla. ${ }^{22}$ The target populations of these interventions were African American women $(n=1)$, Hispanic women $(n=2)$, African American and Hispanic women $(n=3)$, and $\mathrm{AI} / \mathrm{AN}$ women $(n=1)$. We did not identify a single randomized trial targeting Asians. One study ${ }^{50}$ did not specify the race/ethnicity of the participants but instead stated that participants were low-income women. The identified interventions included PC $(n=4)$, professional support $(n=1)$, and enhanced breastfeeding programs $(n=2)$. The details of each randomized trial are summarized in Table 3 . We used the evidence rating scale from the Centre for EvidenceBased Medicine, Oxford, United Kingdom, ${ }^{51}$ to evaluate the quality of each study included in our review. Evidence rating codes can be found in Table 3 . 
Table 3. Summary of Randomized Controlled Trials Evaluating Breastfeeding Interventions TARgeting Racial and ETHNic Minority Women

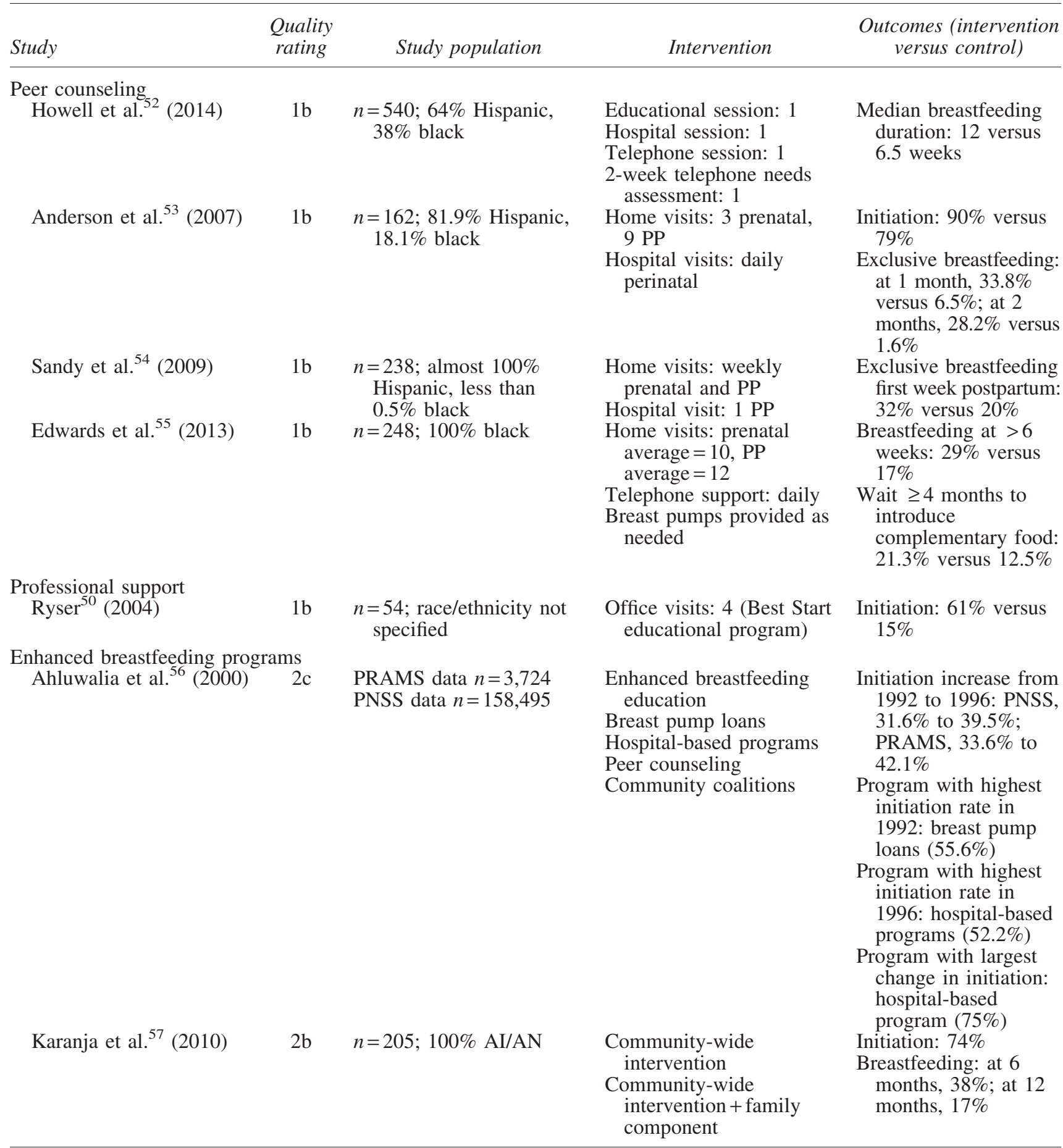

Quality Rating Scale ${ }^{51}$ : $1 \mathrm{~b}=$ individual randomized controlled trials, $2 \mathrm{~b}=$ individual cohort study or low-quality randomized controlled trials, and $2 \mathrm{c}=$ outcomes research; ecological studies.

AI/AN, American Indian/Alaska Native; PNSS, Pregnancy Nutrition Surveillance System; PP, postpartum; PRAMS, Pregnancy Risk Assessment Monitoring System.

$P C$

Four randomized controlled trials evaluating PC interventions among African American and Hispanic women were identified (Table 3). ${ }^{52-55}$ These studies included prenatal, perinatal, and postpartum behavioral education inter- ventions delivered in hospitals or homes or via telephone by social workers, family support workers, and doulas. The intervention programs aimed to prepare and educate mothers about the benefits and challenges of breastfeeding. Overall findings indicate that PC interventions are successful in increasing breastfeeding initiation, duration, and exclusivity 
among minority mothers. Results also suggest that mothers who receive PC are significantly more likely to delay the introduction of complementary foods longer than mothers who receive standard care.

\section{Professional support}

One study evaluated the effectiveness of exposure to the Best Start educational program (versus no exposure to the Best Start program) delivered by an obstetrician-gynecologist (ob-gyn) (Table 3). ${ }^{50}$ Results indicated that mothers $(61 \%)$ who were exposed to the Best Start program had higher rates of breastfeeding initiation than controls $(15 \%)$ at the time of the first week postpartum telephone call $(p=0.01)$. Mothers in the intervention group also exhibited greater breastfeeding intention and positive breastfeeding sentiment. Findings from the review by Chapman and PérezEscamilla $^{22}$ of professional support yielded mixed results; however, none of the studies in their review included interventions delivered by a physician. Given the limited and mixed evidence, current findings should be interpreted with caution, and future research is needed to clarify these results.

\section{Enhanced breastfeeding programs}

Two studies ${ }^{56,57}$ were identified evaluating the effectiveness of enhanced breastfeeding programs. Supporting the findings of Chapman and Pérez-Escamilla, ${ }^{22}$ results from one study $^{56}$ demonstrated that enhanced hospital practices and WIC-based services can have a significant impact on breastfeeding initiation rates. It should be noted that this study analyzed preexisting data from the 1992-1996 Pregnancy Nutrition Surveillance System and the Pregnancy Risk Assessment Monitoring System and may not be representative of the current impact of enhanced breastfeeding programs. Another weakness of this study is the difficulty of evaluating the efficacy of the intervention programs implemented because comparative statistics were not reported. Results from this study indicated that the most successful interventions were hospital-based programs, PC, enhanced breastfeeding education, and breast pump loans, with the hospital-based intervention having the largest change in breastfeeding initiation $(75 \%)$

The second study ${ }^{57}$ identified that implemented an enhanced breastfeeding intervention was also the only study found targeting AI/AN women. The main purpose of this study was to investigate the feasibility of delivering a community-wide intervention, alone or in combination with a family-based intervention, among three AI/AN tribes to promote breastfeeding and reduce the intake of sugarsweetened beverages. Findings illustrated an increase in breastfeeding initiation and duration among all tribes compared with national rates for AI/AN mothers. The rate of breastfeeding initiation among all tribes was $74 \%$, and the rate of breastfeeding at 6 and 12 months was $38 \%$ and $17 \%$, respectively.

\section{Limitations}

An overall limitation to the empirical research reviewed in this analysis is the wide range in the dates when the interventions were conducted. Breastfeeding initiation has increased in the African American community, whereas continuation rates through 6 months among all women have generally increased over the past decade (Figs. 1 and 2). This makes direct comparisons of breastfeeding rates between studies problematic; this is especially true for the one study based on data from the 1990s. ${ }^{56}$ The breastfeeding initiation rates for African American and AI/AN mothers remain significantly below the Healthy People 2020 goal, as does breastfeeding at 6 months for all groups.

\section{Ob-Gyns and Their Patients' Breastfeeding Practices}

Encouraging breastfeeding, especially exclusive breastfeeding, is an area in which ob-gyns can contribute to improving health among women and infants. Ob-gyns are generally supportive of breastfeeding, and the American College of Obstetricians and Gynecologists strongly supports breastfeeding for feeding infants and recommends exclusive breastfeeding for the first 6 months of life. ${ }^{58} \mathrm{Al}-$ though most ob-gyns encourage breastfeeding, there are varying levels of support and promotion among ob-gyn practices. ${ }^{59}$ Findings from a recently published article indicate that breastfeeding conversations between physicians and their patients are infrequent (29\% of visits) and extremely brief (mean of 39 seconds). ${ }^{60}$ Results also revealed that ob-gyn residents were least likely to discuss breastfeeding with their patients compared with certified nurse midwives and nurse practitioners.

In a recent national study, Queenan et al. ${ }^{59}$ examined whether ob-gyns' practices might contribute to breastfeeding rates. Physician reports of breastfeeding rates within their patient populations were consistent with Centers for Disease Control and Prevention rates for states with high, medium, and low rates. Physicians who practiced in states with high breastfeeding rates were more satisfied with their patients' breastfeeding behaviors than were ob-gyns who practiced in states with medium or low rates. Physicians with high proportions of African American or low-income patients reported lower rates of breastfeeding initiation or continuation at 3,6 , or 12 months and were less satisfied with breastfeeding rates among their patients. Physician effort toward encouraging breastfeeding was not associated with their patients' breastfeeding behaviors.

These results suggest that patient demographics, not physician practices, predict low breastfeeding rates. Although Queenan et al. ${ }^{59}$ found that ob-gyn practices do not predict low rates, results indicate that there is still room for improvement among ob-gyns. For example, 55\% of ob-gyns surveyed agreed that formula feeding is an acceptable option that will not harm the infant. Female physicians were significantly more likely than males to indicate that they strongly agree that exclusive breastfeeding is the best option $(68.5 \% \pm 6.0 \%$ versus $44.4 \% \pm$ $6.4 \% ; p=0.005)$.

\section{Ob-Gyn Multicultural Competence to Help Improve Breastfeeding Rates Among Minority Women}

It is important to consider how varying cultural beliefs may influence a woman's perceptions and attitudes toward breastfeeding. Research shows that support and strategies for breastfeeding, particularly targeted at low-income women, can increase breastfeeding initiation and duration. ${ }^{4}$ Given the research documenting frequent barriers to breastfeeding 
Table 4. National Campaigns to Promote Breastfeeding Among Racial and Ethnic Minority Women

\begin{tabular}{ll}
\hline Project title & \\
\hline The Interconception Care Project for California (the College) & Web site \\
& $\begin{array}{c}\text { www.acog.org/About_ACOG/News_Room/News_ } \\
\text { Releases/2012/Outstanding_Contributions } \\
\text { www.babyfriendlyusa.org }\end{array}$ \\
$\begin{array}{l}\text { Best Fed Beginnings } \\
\text { Los Angeles: Managing Obesity in Moms (LA MOMs) }\end{array}$ & $\begin{array}{l}\text { httpublichealth.lacounty.gov/mch/ } \\
\text { Soul Food for Your Baby } \\
\text { The National Hispanic Medical Association }\end{array}$ \\
& $\begin{array}{c}\text { www.nhmamd.org/index.php/programs/obesity- } \\
\text { and-diabetes-initiative }\end{array}$ \\
\hline
\end{tabular}

reported by minority women, ob-gyns should directly address mothers' breastfeeding concerns within a cultural context while exhibiting cultural competence and sensitivity.

Bai et al. ${ }^{61}$ found that the strongest predictor of intention to continue exclusive breastfeeding for Hispanic women was perceived behavioral control. Latina mothers reported that ease of pumping breast milk provided them with a sense of control that pushed them to continue exclusive breastfeeding for 6 months. Bai et al. ${ }^{61}$ recommended that in order "to enable Latina mothers to maintain EBF [exclusive breastfeeding] for 6 months, health professionals need to provide a safe environment to learn and practice pumping, which may entail (a) a provision of electronic or manual breast pump kits at low/reduced prices or free of charge to low income families, (b) less restrictive eligibility criteria for rental electric or manual pumps for WIC participants, (c) preparation of written and visual education materials in Spanish (e.g., pumping/latching demonstration videos, information on proper storage and feeding of expressed milk), and (d) an increased number of Spanish-speaking peer counselors and lactation consultants."

Negative perceptions of breastfeeding in the African American community may have a direct influence on an African American woman's decision to breastfeed. Bai et al. ${ }^{61}$ found that the strongest predictor of intention to continue exclusive breastfeeding for African American women was subjective norm. Results indicated that support from family and friends was very influential in an African American woman's decision to continue exclusive breastfeeding. Ob-gyns should consider including family members, fathers, and close friends of African American mothers in educational discussions about breastfeeding. Family, peer, and father counseling and support groups may also help increase rates for exclusive breastfeeding duration among African American mothers.

Lu et al. ${ }^{62}$ examined racial/ethnic disparities associated with attendance of childbirth classes and the association between attendance and breastfeeding initiation. White women were twice as likely as African American mothers to have ever attended a childbirth class. Mothers who attended childbirth classes were $75 \%$ more likely to initiate breastfeeding than mothers who did not attend. Given the success of childbirth classes and the low attendance among minority women, ob-gyns can help encourage these mothers to attend classes and provide them with appropriate resources for successful attendance. Ideas generated by a focus group of African American women to improve breastfeeding rates included support groups, one-on-one support and mentoring after birth, increased support from healthcare providers, and a social marketing campaign that depicts positive images of African American mothers breastfeeding. ${ }^{39}$

In the Blueprint for Action on Breastfeeding, the Surgeon General discusses the importance of family and community support for minority women during prenatal and postnatal periods. ${ }^{63}$ The report advocates for the promotion and support of breastfeeding continuation upon a mother's return to work, workplaces that facilitate on-site breastfeeding or breast milk expression in private areas, and flexible work hours and breaks. Above all, the report addresses the importance of the healthcare system and society as a whole to send the message to mothers that breastfeeding is completely normal, optimal, nutritional, desirable, and achievable. The Surgeon General advocates for a national public marketing campaign that can work toward obtaining this goal. The Surgeon General's most recent statement, The Surgeon General's Call to Action to Support Breastfeeding, lays out 20 action items and sample implementation strategies for each intervention to increase national breastfeeding rates. ${ }^{63} \mathrm{~A}$ few national breastfeeding campaigns are listed in Table 4.

\section{Closing Comments}

Major efforts are still needed to improve breastfeeding initiation and duration rates in the United States. Research shows that some racial/ethnic minority women face greater obstacles establishing and maintaining breastfeeding. Obgyns have a unique opportunity to promote and support breastfeeding through their clinical practices and public policy. Ob-gyns should be familiar and knowledgeable about breastfeeding recommendations that are laid out in the Guidelines for Perinatal Care by the American Academy of Pediatrics and The American College of Obstetricians and Gynecologists. ${ }^{64}$ Discussions of breastfeeding should begin early during prenatal care. Research shows that women's predelivery intentions are strong predictors of both initiating and continuing breastfeeding. ${ }^{4}$ Ob-gyns are encouraged to begin providing patients with breastfeeding education during the first prenatal appointment and to use all subsequent visits as an opportunity to promote and encourage breastfeeding. ${ }^{58,65}$ Ob-gyns should not only counsel expectant mothers on the positive benefits of breastfeeding, but also discuss possible obstacles that may arise during breastfeeding and strategies to overcome them. In practices with greater racial/ethnic minority populations, early breastfeeding conversations may be even more crucial because these women are more likely to have inconsistent prenatal care. ${ }^{66,67}$ 
The American Academy of Pediatrics and The American College of Obstetricians and Gynecologists advise that supplemental formula should not be given to breastfeeding infants unless otherwise advised by a healthcare professional. ${ }^{64}$ If the infant becomes partially satiated by formula supplementation, the infant will take less milk from the breast, and thus the mother's milk production will decrease. As a result, formula supplementation may significantly reduce the success of breastfeeding.

Ob-gyns' efforts to encourage and support breastfeeding can positively impact breastfeeding practices among women. It is important to first understand and address cultural and social predictors impacting low breastfeeding rates among minority women. These factors influence a woman's breastfeeding attitudes, her decision to breastfeed, and, ultimately, health outcomes for the mother and child. By directly addressing these obstacles and promoting positive breastfeeding practices, ob-gyns can have a meaningful impact on the future health of the mother and child.

\section{Acknowledgments}

Financial support for this study was provided in part by grant UA6MC1901 0 from the Maternal and Child Health Bureau (Title V, Social Security Act, Health Resources and Services Administration, and U.S. Department of Health and Human Services).

\section{Disclosure Statement}

No competing financial interests exist.

\section{References}

1. American Academy of Pediatrics Section on Breastfeeding. Breastfeeding and the use of human milk. Pediatrics 2005;115:496-506.

2. Centers for Disease Control and Prevention. Racial and ethnic differences in breastfeeding initiation and duration, by state-National Immunization Survey, United States, 2004-2008. Morb Mortal Wkly Rep 2010;59:327-334.

3. Centers for Disease Control and Prevention. Provisional breastfeeding rates by socio-demographic factors, among children born in 2007. Available at www.cdc.gov/breast feeding/data/NIS_data/2007/socio-demographic_any.htm (accessed August 31, 2013).

4. Ahluwalia IB, Morrow B, Hsia J. Why do women stop breastfeeding? Findings from the Pregnancy Risk Assessment and Monitoring System. Pediatrics 2005;116:14071412.

5. Forste R, Weiss J, Lippincott E. The decision to breastfeed in the United States: Does race matter? Pediatrics 2001; 108:291-296.

6. Hawkins SS, Stern AD, Gillman MW. Do state breastfeeding laws in the USA promote breast feeding? J Epidemiol Community Health 2013;67:250-256.

7. Centers for Disease Control and Prevention. Progress in increasing breastfeeding and reducing racial/ethnic differences-United States, 2000-2008 births. Morb Mortal Wkly Rep 2013;62:77-80.

8. Finer LB, Henshaw SK. Disparities in rates of unintended pregnancy in the United States, 1994 and 2001. Perspect Sex Reprod Health 2006;38:90-96.
9. American College of Obstetricians and Gynecologists. Breastfeeding in underserved women: Increasing initiation and continuation of breastfeeding. Committee Opinion No. 570. Obstet Gynecol 2013;122:423-428.

10. Mirsa D, ed. The Women's Health Data Book: A Profile of Women's Health in the United States, 3rd ed. Washington, DC: Jacobs Institute of Women's Health and The Henry J. Kaiser Family Foundation, 2001.

11. American Heart Association. Heart disease and stroke statistics-2012 update: A report from the American Heart Association. 2010. Available at www.americanheart.org (accessed August 20, 2012).

12. Kruain AK, Cardarelli KM. Racial and ethnic differences in cardiovascular disease risk factors: A systemic review. Ethn Dis 2007;17:143-152.

13. Jarlenski MP, Bennett WL, Bleich SN, et al. Effects of breastfeeding on postpartum weight loss among U.S. women. Prev Med 2014;69:146-150.

14. Hatsu IE, McDougald DM, Anderson AK. Effect of infant feeding on maternal body composition. Int Breastfeed $J$ 2008;3:18.

15. Harder T, Bergmann R, Kallischnigg G, et al. Duration of breastfeeding and risk of overweight: A meta-analysis. Am $J$ Epidemiol 2005;162:397-403.

16. Steube AM, Rich-Edwards JW, Willet WC, et al. Duration of lactation and incidence of type 2 diabetes. JAMA 2005;294:2601-2610.

17. Schwarz EB, Ray RM, Stuebe AM, et al. Duration of lactation and risk factors for maternal cardiovascular disease. Obstet Gynecol 2009;113:974-982.

18. Stuebe AM, Schwarz EB, Grewen K, et al. Duration of lactation and incidence of maternal hypertension: A longitudinal cohort study. Am J Epidemiol 2011;174:11471158.

19. U.S. Department of Health and Human Services. Healthy People 2020 topics and objectives: Family planning. Available at http://healthypeople.gov/202/topicsobjectives 2020/overview.aspx?topicid $=13$ (accessed December 1 , 2014).

20. Kennedy KI, Visness CM. Contraceptive efficacy of lactational amenorrhoea. Lancet 1992;339:227-230.

21. Ramos DE. Breastfeeding: A bridge to addressing disparities in obesity and health. Breastfeed Med 2012;7:354-357.

22. Chapman DJ, Pérez-Escamilla R. Breastfeeding among minority women: Moving from risk factors to interventions. Adv Nutr 2012;3:95-104.

23. Taveras EM, Gillman MW, Kleinman K, et al. Racial/ ethnic differences in early-life risk factors for childhood obesity. Pediatrics 2010;125:686-695.

24. Chapman DJ, Pérez-Escamilla R. US national breastfeeding monitoring and surveillance: Current status and recommendations. J Hum Lact 2009;25:139-150.

25. Pérez-Escamilla R, Putnik P. The role of acculturation in nutrition, lifestyle, and incidence of type 2 diabetes among Latinos. J Nutr 2007;137:860-870.

26. Chapman DJ, Damio G, Young S, et al. Effectiveness of breastfeeding peer counseling in a low-income, predominantly Latina population. Arch Pediatr Adolesc Med 2004;158:897-902.

27. Beck CT. Acculturation: Implications for perinatal research. Am J Matern Child Nurs 2006;31:114-120.

28. Rassin DK, Markides KS, Baranowski T, et al. Acculturation and the initiation of breastfeeding. J Clin Epidemiol 1994;47:739-746. 
29. Kimbro RT, Lynch SM, McLanahan S. The Hispanic paradox and breastfeeding: Does acculturation matter? Evidence from the Fragile Families Study. 2004. Available at http://crcw.princeton.edu/workingpapers/WP04-01-FF-Kimbro .pdf (accessed August 6, 2013).

30. Romero-Gwynn E, Carias L. Breast-feeding intentions and practice among Hispanic mothers in southern California. Pediatrics 1989;84:626-632.

31. Singh GK, Kogan MD, Dee DL. Nativity/immigrant status, race/ethnicity, and socioeconomic determinants of breastfeeding initiation and duration in the United States, 2003. Pediatrics 2007;119(Suppl 1):S38-S46.

32. Anderson AK, Damio G, Himmelgreen DA, et al. Social capital, acculturation, and breastfeeding initiation among Puerto Rican women in the United States. J Hum Lact 2004;20:39-45.

33. Sussner KM, Lindsay AC, Peterson KE. The influence of acculturation on breast-feeding initiation and duration in low-income women in the US. J Biosoc Sci 2008;40:673696.

34. Lindberg SM, Adams AK, Prince RJ. Early predictors of obesity and cardiovascular risk among American Indian children. Matern Child Health J 2012;16:18791886.

35. Li R, Darling N, Maurice E, et al. Breastfeeding rates in the United States by characteristics of the child, mother, or family: The 2002 National Immunization Survey. Pediatrics 2005;115:e31-e37.

36. Sparks PJ. Racial/ethnic differences in breastfeeding duration among WIC-eligible families. Womens Health Issues 2011;21:374-382.

37. Carmichael S, Prince C, Burr R, et al. Breast-feeding practices among WIC participants in Hawaii. J Am Diet Assoc 2001;101:57-62.

38. Hurst CG. Addressing breastfeeding disparities in social work. Health Soc Work 2007;32:207-210.

39. Kulka TR, Jensen E, McLaurin S, et al. Community based participatory research of breastfeeding disparities in African American women. Infant Child Adolesc Nutr 2011; $3: 233-239$

40. Nommsen-Rivers LA, Chantry CJ, Cohen RJ, et al. Comfort with the idea of formula feeding helps explain ethnic disparity in breastfeeding intentions among first-time mothers. Breastfeed Med 2010;5:25-33.

41. Flower KB, Willoughby M, Cadigan RJ, et al. Understanding breastfeeding initiation and continuation in rural communities: A combined qualitative/quantitative approach. Matern Child Health 2008;12:402-414.

42. Beal AC, Kuhlthau K, Perrin JM. Breastfeeding advice given to African American and white women by physicians and WIC counselors. Public Health Rep 2003;118:368376.

43. U.S. Department of Agriculture. WIC participant and program characteristics 2010, WIC-1 PC. 2011. Available at www.fns.usda.gov/sites/default/files/WICPC2010.pdf (accessed August 31, 2013).

44. Asiodu I, Flaskerud JH. Got milk? A look at breastfeeding from an African American perspective. Issues Ment Health Nurs 2011;32:544-546.

45. Lu MC, Lange L, Slusser W, et al. Provider encouragement of breast-feeding: Evidence from a national survey. Obstet Gynecol 2001;97:290-295.

46. Colen CG, Ramey DM. Is breast truly best? Estimating the effects of breastfeeding on long-term child health and wellbeing in the United States using sibling comparisons. Soc Sci Med 2014;109:55-65.

47. Galtry J, Callister P. Assessing the optimal length of parental leave for child and parental well-being: How can research inform policy? J Fam Issues 2005;26:219-246.

48. Satcher DS. DHHS blueprint for action on breastfeeding. Public Health Rep 2001;116:72-73.

49. World Health Organization. Infant and Young Child Nutrition: Global Strategy on Infant and Young Child Feeding, Vol. A55. Geneva: World Health Organization, 2002.

50. Ryser FG. Breastfeeding attitudes, intention, and initiation in low-income women: The effect of the Best Start program. J Hum Lact 2004;20:300-305.

51. Centre for Evidence-Based Medicine. Levels of evidence. 2014. Available at www.essentialevidenceplus.com/product/ ebm_loe.cfm?show $=$ oxford $($ accessed October 15, 2014).

52. Howell EA, Bodnar-Deren S, Balbierz A, et al. An intervention to extend breastfeeding among black and Latina mothers after delivery. Am J Obstet Gynecol 2014;210: 39.e1-e5.

53. Anderson AK, Damio G, Chapman DJ, et al. Differential response to an exclusive breastfeeding peer counseling intervention: The role of ethnicity. J Hum Lact 2007;23:16-23.

54. Sandy JM, Anisfeld E, Ramirez E. Effects of a prenatal intervention on breastfeeding initiation rates in a Latina immigrant sample. J Hum Lact 2009;25:404-411.

55. Edwards RC, Thullen MJ, Korfmacher J, et al. Breastfeeding and complementary food: Randomized trial of community doula home visiting. Pediatrics 2013;132(Suppl 2): S160-S66.

56. Ahluwalia IB, Tessaro I, Grummer-Strawn LM, et al. Georgia's breastfeeding promotion program for low-income women. Pediatrics 2000;105:e85-e90.

57. Karanja N, Lutz T, Ritenbaugh C, et al. The TOTS community intervention to prevent overweight in American Indian toddlers beginning at birth: A feasibility and efficacy study. J Community Health 2010;35:667-675.

58. American College of Obstetricians and Gynecologists. Special Report from ACOG. Breastfeeding: Maternal and infant aspects. ACOG Clin Rev 2007;12:1S-16S.

59. Queenan J, Power ML, Farrow V, et al. U.S. obstetriciangynecologists' estimates of their patients' breastfeeding rates. Obstet Gynecol 2012;119:838-844.

60. Demirci JR, Bogen DL, Holland C, et al. Characteristics of breastfeeding discussions at the initial prenatal visit. Obstet Gynecol 2013;122:1263-1270.

61. Bai Y, Wunderlich SM, Fly AD. Predicting intentions to continue exclusive breastfeeding for 6 months: A comparison among racial/ethnic groups. Matern Child Health $J$ 2011;15:1257-1264.

62. Lu MC, Prentice J, Yu SM, et al. Childbirth education classes: Sociodemographic disparities in attendance and the association of attendance with breastfeeding initiation. Matern Child Health J 2003;7:87-93.

63. McGuire S. The Surgeon General's Call to Action to Support Breastfeeding. Adv Nutr 2011;2:523-524.

64. American Academy of Pediatrics, American College of Obstetricians and Gynecologists. Guidelines for Perinatal Care, 7th ed. Elk Grove Village, IL: American Academy of Pediatrics; and Washington, DC: American College of Obstetricians and Gynecologists, 2012.

65. Queenan JT. Academy of Breastfeeding Medicine Founder's Lecture 2010: Breastfeeding: An obstetrician's view. Breastfeed Med 2011;6:7-14. 
66. Feijen-de Jong EI, Jansen DE, Baarveld F, et al. Determinants of late and/or inadequate use of prenatal healthcare in high-income countries: A systematic review. Eur J Public Health 2012;22:904-913.

67. Partridge S, Balayla J, Holcroft CA, et al. Inadequate prenatal care utilization and risks of infant mortality and poor birth outcome: A retrospective analysis of 28,729,765 U.S. deliveries over 8 years. Am J Perinatol 2012;29:787-794.
Address correspondence to: Michael L. Power, PhD

Department of Research American College of Obstetricians and Gynecologists 409 12th Street, SW Washington, DC 20024

E-mail: mpower@acog.org 